\title{
ON THE INNER AUTOMORPHISMS OF A COMPACT GROUP
}

\author{
SHINGO MURAKAMI and MORIKUNI GOTÔ
}

In this note, we give a characterization of inner automorphisms in the set of automorphisms of a compact connected group, and then apply it to give a proof of a theorem due to $K$. Iwasawa on the group of automorphisms of a compact group."

Lemma. Let $G$ be a compact connected Lie gronp and $\tau$ an automorphism of $G$. Assume that $\tau$ transforms every element of $G$ to its conjugate element. Then $\tau$ is an inner automorphism.

Proof. Let $Z$ be the center of $G$ and $S$ the maximal semi-simple subgroup of $G$. Then $Z$ and $S$ are characteristic subgroups and $G=Z S$. Under our assumption, $\tau$ leaves fixed every element of $Z$ and induces in $S$ an automorphism satisfying the same assumption in $S$ as the one for $\tau$ in $G$. From these, it is easily seen that the lemma is reduced to the case where the group $G$ is semisimple.

For a semi-simple compact connected Lie group $G$, the following facts are known ${ }^{2)}$ and will be used later. The maximal abelian subgroups are connected and they are mutually conjugate. Let $T$ be one of them and $l$ its dimension. The Lie algebra $\mathfrak{S}$ of $T$ is isomorphic to the $l$-dimensional vector group and the exponential mapping; $h \rightarrow \exp h{ }^{3)} h \in \mathfrak{S}$, is a homomorphism of $\mathfrak{S}$ onto $T$ with discrete kernel $\Re$. The root forms $\alpha_{1}, \ldots, \alpha_{m}$ of $G$ may be defined as the real-valued linear forms on $\mathfrak{g}$ for which $\sqrt{-1} \alpha_{i}(h)(i=1, \ldots, m)$ are the eigenvalues of the adjoint mapping of an element $h$ of $\mathscr{S}$ in the Lie algebra of $G$. $\mathscr{S}$ is regarded as a euclidean space with respect to the positive definite quadratic form $\varphi(h)=\sum_{i=1}^{m}\left(\alpha_{i}(h)\right)^{2}$. For a root $\alpha_{i}$, we denote by $E_{i}$ the $(l-1)$ dimensional linear subspace defined by $\alpha_{i}(h)=0$. Now an automorphism $\tau$ of $G$ with the property $\tau(T)=T$ induces an orthogonal linear transformation $\bar{\tau}$ in $\mathfrak{S}$ satisfying the following relation:

Received November 12, 1951.

1) These theorems were first proved by the second author several years ago in a different way.

2) See e.g. [4].

3) exp $h$ denotes the point $h(1)$ of the one-parameter subgroup $h(t)$ with the tangential vector $h$ at the identity. 


$$
\tau(\exp h)=\exp (\bar{\tau}(h)), \quad h \in \mathfrak{g} .
$$

All such $\bar{\tau}$ 's form a group $\mathfrak{T} . \bar{\tau}$ is the identical transformation if and only if $\tau$ is an inner automorphism raised up by an element of $T . \bar{\tau}$ is induced by an inner automorphism of $G$, if and only if it belongs to the linear transformation group $\subseteq$ generated by the reflections with respect to $E_{i}(i=1, \ldots, m)$. Let $\mathfrak{S}_{0}=\mathfrak{S}_{2}-\bigcup_{i=1}^{m} E_{i}$. Then the connected components of $\$_{0}$ are permuted among themselves by the transformations of $\mathfrak{T}$, and transitively even by those of $\subseteq$. Morenver, let $\Pi$ be any one of these connected components of $\mathscr{S}_{0}$. Then $\Pi$ is a fundamental domain of $\mathfrak{S}$ and it contains a polyhedral domain $P$ of which boundary contains the origin and which is a fundamental domain of the linear transformation group on $\mathfrak{S}$ generated by the transformations of $\subseteq$ and by translations by the elements of $\Re$. This domain $P$ is defined as follows: Let $E_{i}(k)$, $k=0, \pm 1, \pm 2, \ldots$, be the hyperplane defined by $\alpha_{i}(h)=k$. In the connected components of $\mathfrak{H}_{i}-\bigcup_{i=1}^{\infty} \bigcup_{k=-\infty}^{\infty} E_{i}(k)$, there exists uniquely ach a component $Q$ which is contained in $I I$ and of which boundary contains the origin $o$. Let $o, p_{1}, p_{2}$, $\ldots, p_{r}$ be the elements of $\Re$ which are contained in the boundary of $Q$. $P$ is the set of all elements $h$ in $Q$ which satisfy the relation

$$
\|o h\|<\min _{1 \leqq i \leqq r}\left\|o p_{i}\right\|
$$

where $\|\cdot\|$ denotes the distance in $\mathfrak{2}$. The subset $\exp P$ in $G$ contains (one and) only one conjugate element of a (regular) element of $G$. We remark that $P$ is transformed by any $\bar{\tau}$ of $\mathfrak{I}$ to the same kind of polyhedral domain with respect to some connected component of $\mathscr{S}_{0}$ and especially $\bar{\tau}(P)=P$ for such $\zeta$ as $\bar{\tau}(\Pi)=\Pi$.

Reserving these notations, we shall prove the lemma for a semi-simple compact connected Lie group. Since $\tau(T)$ is a maximal abelian subgroup of $G$, there is an inner automorphism $\sigma_{1}$ such that $\sigma_{1} \tau(T)=T$. Then we can consider the linear transformation $\overline{\sigma_{1} \tau}$ on $\mathscr{\&}$. $\overline{\sigma_{1} \tau}$ transforms $I I$ to another connected component $\Pi^{\prime}$ of $\mathscr{J}_{0}$. There exists a transformation belonging to $\Xi$ which maps $\Pi^{\prime}$ onto $\Pi$. In other words, there is an inner automorphism $\sigma_{2}$ such that $\sigma_{2}(T)=T$ and that $\bar{\sigma}_{2}\left(\Pi^{\prime}\right)=\Pi$. Set $\tau^{\prime}=\sigma_{2} \sigma_{1} \tau$. It is clear that $\tau^{\prime}(T)=T$ and $\bar{\tau}^{\prime}(I)=\Pi$. We shall show that under our assumption on $\tau \bar{\tau}^{\prime}$ is the identical transformation and therefore $\tau^{\prime}$ is an inner automorphism, which will prove our lemma. Since $\bar{\tau}^{\prime}(I)=\Pi, \bar{\tau}^{\prime}(P)=P$ and hence $\bar{\tau}^{\prime}(h) \in P$ for $h \in P$. On the other hand, since $\tau^{\prime}$ satisfies the same assumption as the one for $\tau, \tau^{\prime}(\exp h)$ $=\exp \left(\bar{\tau}^{\prime}(h)\right)$ is conjugate to $\exp h$. Thus by the properties of $P$, we must have $\bar{\tau}^{\prime}(h)=h$ for $h \in P$. Because $P$ is an open domain and $\bar{\tau}^{\prime}$ is a linear transformation, $\bar{\tau}^{\prime}$ is the identical transformation.

THEOREM 1. Let $G$ be a compact connected group. An automorphism $\tau$ is 
an inner automorphism, if (and obviously only if) it transforms every element of $G$ to its conjugate element.

Proof. There exists a family $\left\{N_{\alpha}\right\}$ of normal subgroups in $G$ such that $G_{\alpha}=G / N_{\alpha}$ are compact connected Lie groups and that $G$ is the $G_{\alpha}$-adic limit group of $\left\{G_{\alpha}\right\} ; G=\lim G_{\alpha}$. By our assumption. $\tau\left(N_{\alpha}\right)=N_{\alpha}$ and hence $\tau$ induces an automorphism $\tau_{\alpha}$ in every group $G_{\alpha}$. It is obvious that $\tau_{\alpha}$ satisfies the same condition in $G_{\alpha}$ as the one for $\tau$ in $G$. Applying the above lemma to $\tau_{\alpha}$ and $G_{\alpha}$, we see that $\tau_{a}$ is an inner automorphism. It follows that there exists a set $M_{*}$ in $G$ such that for any $y \in M_{\alpha}$

$$
\tau(x) \equiv y^{-1} x y \quad \bmod . N_{\alpha} .
$$

Then. it is easily verified that the family of sets $\left\{M_{\alpha}\right\}$ has the finite intersection property. As $G$ is compact. there exists an element $y \in \bigcap_{a} M_{\alpha}$, for which we have

$$
\because(x) \equiv y^{-1} x y \quad \bmod . N_{\alpha} \text { for all } \alpha,
$$

hence

$$
\tau(x)=y^{-1} x y .
$$

This proves that $r$ is an inner automorphism.

Remark. This theorem is not true without connectedness of the group, as was shown by W. Burnside [2].

By Peter-Weyl's theorem. for any two elements $x$ and $y$ which are not conjugate to one another, there exists a representation of the group such that for its character $\chi$ we have $\chi(x) \neq \chi(y)$. From this, it is easily seen that Theorem 1 is equivalent to the following

THEOREM 1'. Let $G$ be a compact connected group and : an automorphism of $G$. If for any representation $D: x \rightarrow D(x), x \in G$, the representation $D^{\tau}$ defined by $D^{\tau}(x)=D(\tau(x))$ is equivalent to $D$, then $\tau$ is an inner automorphism.

Ramark. In this form the theorem has been proved by M. Abe [1], making use of the classification of simple Lie algebras.

Le: $G$ be a compact group and $A(G)$ the group of all automorphisms of $G$. $A(G)$ is topologized by taking as the neighborhoods of the identity the sets of the following form;

$$
U(F, V)=\{r ; r \in A(G),:(x) \subset x V \text { for all } x \in F\},
$$

where $t$ and $V$ are respectively any compact set and any one of the neighborhoods of the identity in $G$. This topology coincides with the one defined as follows. For any finite number of complex-valued continuous functions $f_{1}, \ldots, f_{n}$ on $G$ and $s>0$. we take as a neighborhood of the identity the set 
$U\left(f_{1}, \ldots, f_{n}: \varepsilon\right)=\left\{\tau ; \tau \in A(G), \max _{x \in G}\left|f_{i}(x)-f_{i}(\tau(x))\right|<\varepsilon\right.$ for $\left.i=1, \ldots, n\right\}$. $I(G)$ denotes the closed normal subgroup of $A(G)$ which consists of all inner automorphisms of $G$.

We shall prove as an application of Theorem $1^{\prime}$ the following theorem of $\mathrm{K}$. Iwasawa ${ }^{4)}$ in the case where the groups under considerations are conneced.

THEOREM 2. Let $G$ be a compact connected group. Then $A(G) / I(G)$ is totally disconnected.

Proof. For the character $\%$ of a representation of $G$, we put

$$
A_{x}=\{\tau ; \tau \in A(G), \chi(\tau(x))=\chi(x) \text { for } x \in G\} .
$$

Then, as was shown in [3] p. 513, $A_{\mathrm{x}}$ is an open subgroup of $A(G)$. Theorem $1^{\prime}$ asserts that $I(G)=\bigcap_{\mathrm{x}} A_{\mathrm{x}}$, where $\%$ runs over the characters of all representations of $G$. This proves the theorem.

Supplementary Remarks to the Proof of Iwasawa's Theorem. Our method in proving this theorem is in some sense a generalization of the one which is used in [3] for the case where the groups are abelian. In the latter case, we may transfer to the dual automorphisms in the dual group. In our case, we have done this transference by means of Theorem $1^{\prime}$ and the automorphisms are considered modulo inner automorphisms as dual transformations between characters. The second topology mentioned above in $A(G)$ may be seen to be the topology for these dual transformations. From these points of view, we might have two hopes. First, we may desire to give a direct proof of Theorem $1^{\prime}$ without any help of the structure of groups so as to make our discussion completely depend on the dual transformations. The writers do not succeed to do so. Secondly, since the original proof in [3] is essentially owing to the structure of groups. it might be desirable that the use of the second topology in it is replaced by that of direct one, i.e., the first topology in $A(G)$ mentioned above. This is done as follows. Keeping the notations in the proofs of Theorems 1 and 2, the point where the second topology is needed is in showing the above quoted fact that $A_{x}$ is an open subgroup. We consider the following subgroup $A_{\alpha}$ instead of $A_{x}$,

$$
A_{\alpha}=\left\{\tau ; \tau \in A(G), \tau\left(N_{\alpha}\right)=N_{\alpha}\right\} \text {. }
$$

Then, since $G_{\alpha}=G / N_{\alpha}$ is a Lie group, there exists a sufficiently small neighborhood $V$ of the identity in $G$ such that $N_{\alpha} V$ does not contain any subgroup larger than $N_{\alpha}$. Let $\sigma$ belong to the neighborhood $U\left(N_{\alpha}, V\right)$ in the first topology of $A(G)$. Then we have $\sigma\left(N_{\alpha}\right) \subset N_{\alpha}$ and, as $G / \sigma\left(N_{\alpha}\right)$ and $G / N_{\alpha}$ are isomorphic Lie groups, $\sigma\left(N_{\alpha}\right)=N_{\alpha}$. This shows that $U\left(N_{\alpha}, V\right) \subset A_{\alpha}$, and therefore that

4) Theorem 1 in [3] p. 509 . 
$A_{\alpha}$ is an open subgroup. We may discuss hereafter just as in the original proof in [3] making use of $A_{\alpha}$ in place of $A_{x}$.

\title{
BIBLIOGRAPHY
}

[1] M. Ake; On Lie algebras with commutative radical. Zenkoku sijô Sûgaku Danwakai. Vol. 226 (1941). (in Japanese).

[2] W. Burnside; On the outer isomorphism of a group. Proc. London Math. Soc. (2) Vol. 11 (1911).

[3] K. Iwasawa; On some types of topological groups. Ann. of Math. Vol. 50 (1949).

[4] H. Weyl; The structure and representations of continuous groups. Lectures at the Institute for Advanced Study, Princeton, 1934-1935.

\author{
Osaka University \\ and \\ University of Education, Tokyo
}

